\title{
An ab initio variationally computed room-temperature line list for ${ }^{32} \mathrm{~S}^{16} \mathrm{O}_{3}$
}

\author{
Daniel S. Underwood, Jonathan Tennyson and Sergei N. Yurchenkd* \\ Department of Physics and Astronomy, \\ University College London, Gower Street, \\ London WC1E 6BT, United Kingdom
}

(Dated: June 16, 2021)

\begin{abstract}
$A b$ initio potential energy and dipole moment surfaces are computed for sulfur trioxide $\left(\mathrm{SO}_{3}\right)$ at the CCSD(T)-F12b level of theory and appropriate triple-zeta basis sets. These surfaces are fitted and used, with a slight correction for the equilibrium S-O distance, to compute pure rotational and rotation-vibraton spectra of ${ }^{32} \mathrm{~S}^{16} \mathrm{O}_{3}$ using the variational nuclear motion program TROVE. The calculations considered transitions in the region $0-4000 \mathrm{~cm}^{-1}$ with rotational states up to $J=85$. The resulting line list of 349348513 transitions is appropriate for modelling room temperature ${ }^{32} \mathrm{~S}^{16} \mathrm{O}_{3}$ spectra. Good agreement is found with the observed infrared absorption spectra and the calculations are used to place the measured relative intensities on an absolute scale. A list of 10878 experimental transitions is provided in a form suitable for inclusion in standard atmospheric and planetary spectroscopic databases.
\end{abstract}

\footnotetext{
${ }^{*}$ To whom correspondence should be addressed: s.yurchenko@ucl.ac.uk
} 


\section{INTRODUCTION}

Sulfur trioxide $\left(\mathrm{SO}_{3}\right)$ is a stable, planar, symmetric molecule whose electronic ground state is a closed shell. On earth it is a pollutant produced from smoke-stacks and other industrial exhausts [1]. In the atmosphere $\mathrm{SO}_{3}$ forms sulfuric acid with its association with acid rain, and inside combustion systems it is corrosive. In both cases the reactivity of $\mathrm{SO}_{3}$ makes it production hard to monitor [2]. $\mathrm{SO}_{3}$ is produced naturally on earth as part of volcanic emissions [3]. It is also thought to be a significant constituent of the atmosphere of Venus [4].

The infrared vibration-rotation spectrum of ${ }^{32} \mathrm{~S}^{16} \mathrm{O}_{3}$ (henceforth referred to as $\mathrm{SO}_{3}$ ) has been extensively investigated in a series of papers by Maki and co-workers [5-11]. Its "forbidden" rotational spectrum, for which centrifugal distortions can induce transitions, has been investigated using microwave Fourier-transform spectroscopy [12]. However, although these works provide an extensive list of measured line frequencies, none of them report absolute transition intensities.

There has been limited theoretical work on $\mathrm{SO}_{3}$. Dorney et al. [13] reported force constants, while Martin [14] computed an ab initio potential energy surface (PES) using coupled cluster methods and reported theoretical estimates for the band origins of the lowlying vibrational states. Again, neither of these works considered transition intensities.

The lack of any absolute transition intensities for $\mathrm{SO}_{3}$ places severe limitations on the use of its infrared spectrum for remote sensing applications or inclusion of this data in standard atmospheric and planetary spectroscopic databases [15, 16]. In this work we report the calculation of a new ab initio PES and associated dipole moment surfaces (DMS) for $\mathrm{SO}_{3}$. These are used to not only produce theoretical spectra for ${ }^{32} \mathrm{~S}^{16} \mathrm{O}_{3}$, but also to place the relative intensity measurements of its infrared spectrum on an absolute scale.

\section{THE POTENTIAL ENERGY AND DIPOLE MOMENT SURFACES}

The ab initio PES was computed using the recently-proposed explicitly correlated F12 singles and doubles coupled cluster method including a perturbational estimate of connected triple excitations, CCSD(T)-F12b [17], in conjunction with the corresponding F12-optimized correlation consistent basis sets, namely the valence correlation-consistent functions aug-cc- 
pVTZ-F12 and aug-cc-pV(T+d)Z-F12 for oxygen and sulfur, respectively [18]. We also utilized the OptRI [19] cc-pV5Z/JKFIT [20] and aug-cc-pwCV5Z/MP2FIT [21] auxiliary basis sets for evaluating the many-electron integrals, the exchange and Fock operators, and the remaining electron repulsion integrals, respectively. The value of the geminal Slater exponent $\beta$ was chosen as 1.2. In ab initio calculations Molpro.2010 [22] was employed. We used a large grid of 13000 points with the stretching modes ranging from 1.1 to $2.0 \AA$, interbond angles $\alpha$ between 70 and $123^{\circ}$, and the inversion angle $\rho$ between 0 and $50^{\circ}$. This grid was sufficient to cover energies up to $40000 \mathrm{~cm}^{-1}$ above the minimum.

This PES was then represented in an analytical form given by the expansion [23]

$$
\begin{aligned}
V\left(\xi_{1}, \xi_{2}, \xi_{3}, \xi_{4 a}, \xi_{4 b} ; \sin \bar{\rho}\right) & =V_{\mathrm{e}}+V_{0}(\sin \bar{\rho})+\sum_{j} F_{j}(\sin \bar{\rho}) \xi_{j} \\
& +\sum_{j \leq k} F_{j k}(\sin \bar{\rho}) \xi_{j} \xi_{k}+\sum_{j \leq k \leq l} F_{j k l}(\sin \bar{\rho}) \xi_{j} \xi_{k} \xi_{l} \\
& +\sum_{j \leq k \leq l \leq m} F_{j k l m}(\sin \bar{\rho}) \xi_{j} \xi_{k} \xi_{l} \xi_{m}
\end{aligned}
$$

in the coordinates $\xi_{k}$ :

$$
\begin{aligned}
\xi_{k} & =1-\exp \left(-a\left(r_{k}-r_{\mathrm{e}}\right)\right), k=1,2,3, \\
\xi_{4 a} & =\frac{1}{\sqrt{6}}\left(2 \alpha_{1}-\alpha_{2}-\alpha_{3}\right), \\
\xi_{4 b} & =\frac{1}{\sqrt{2}}\left(\alpha_{2}-\alpha_{3}\right), \\
\sin \bar{\rho} & =\frac{2}{\sqrt{3}} \sin \left[\left(\alpha_{1}+\alpha_{2}+\alpha_{3}\right) / 6\right],
\end{aligned}
$$

where

$$
V_{0}(\sin \bar{\rho})=\sum_{s=1} f_{0}^{(s)}\left(\sin \rho_{e}-\sin \bar{\rho}\right)^{s}
$$

and

$$
F_{j k \ldots}(\sin \bar{\rho})=\sum_{s=0} f_{j k \ldots}^{(s)}\left(\sin \rho_{e}-\sin \bar{\rho}\right)^{s} .
$$

The same form has been used to represent the PESs of $\mathrm{NH}_{3}, \mathrm{PH}_{3}, \mathrm{SbH}_{3}, \mathrm{NH}_{3}{ }^{+}$, and $\mathrm{BiH}_{3}$ [24 27]. The potential parameters $f_{j k \ldots}^{(s)}$ were obtained through a least squares fit to the $a b$ initio points with an root-mean-squares (rms) error of $0.067 \mathrm{~cm}^{-1}$. Weight factors were set using the expression suggested by Partridge and Schwenke [28]:

$$
w_{i}=\frac{\tanh \left[-0.0005 \mathrm{~cm} \times\left(V_{i}-16000 \mathrm{~cm}^{-1}\right)\right]+1.002002002}{2.002002002 \mathrm{~cm} \times V_{i}^{(w)}},
$$


where $V_{i}^{(w)}=\max \left(16000 \mathrm{~cm}^{-1}, V_{i}\right)$, and $V_{i}$ is the ab initio energy at the $i$ th geometry (in $\mathrm{cm}^{-1}$ ), measured relative to the equilibrium energy. The ab initio energy $V_{i}$ is weighted by the factor $w_{i}$ in the PES fitting; these weight factors favor the energies below 16000 $\mathrm{cm}^{-1}$. The $a b$ initio equilibrium geometry $r_{\mathrm{e}}$ as obtained from the fitting is $1.42039 \AA$. This can be compared with an experimentally derived value $1.41732 \AA[6]$. We found that the experimental rotational energies of $\mathrm{SO}_{3}$ [8] are best described by the latter value. Therefore we decided to use this value of $r_{\mathrm{e}}$ in all our calculations in place of the $a b$ initio value. It is known however that replacing the equilibrium structure may cause undesirably large changes to the ro-vibrational energies. In order to minimize this effect the following procedure was employed.

The idea is to expand the ab initio PES around some reference value $r_{\text {ref }}$ and than remove the linear terms in the PES expansion and replace the expansion center with $r_{\mathrm{e}}^{(\exp )}$. In doing this we expect the change of the shape in the PES to partly compensate the effect from replacing $r_{\mathrm{e}}^{(\mathrm{ai})}$ with $r_{\mathrm{e}}^{(\mathrm{exp})}$ on the vibrational energies. We choose the reference center $r_{\mathrm{e}}^{(\mathrm{ref})}=1.42039+\Delta r_{\mathrm{e}}$, where $\Delta r_{\mathrm{e}}=r_{\mathrm{e}}^{(\mathrm{ai})}-r_{\mathrm{e}}^{(\exp )}=0.00307$, i.e. on the opposite side from $r_{\mathrm{e}}^{(\exp )}$ and at the same distance from $r_{\mathrm{e}}^{(\exp )}$.

This is the only adjustment to the shape of ab initio PES utilized. Table I compares rotational levels obtained by Maki et al. [8] and those computed using the equilibriumadjusted PES used in this work. The agreement is very good.

The DMS were calculated using the same level of theory as the PES and on the same grid of 13000 points. The ab initio values were then expressed analytically using the symmetrized molecular bond (SMB) representation described in detail in Ref. [29]. The resulting dipole moment parameters obtained through a least squares fit reproduce the $a b$ initio data with an rms error of 0.00013 D. In these fittings the same factors defined by Eq. (8) were used to weight the geometries according to the corresponding energies. To our knowledge there are no experimental or $a b$ initio dipole moment data in the literature that we could use to validate our DMS against. However, our experience of working dipole moments for different systems [29 32] shows that ab initio intensities in most cases are competitive with experimental measurements. The quality of the relative intensities calculated using our DMS is discussed below.

Both the potential energy and dipole moments functions used in the present work are given as supplementary data. 
TABLE I: Theoretical rotational term values for ${ }^{32} \mathrm{~S}^{16} \mathrm{O}_{3}$ ground vibrational state $\left(\mathrm{cm}^{-1}\right)$ compared with experiment [8].

\begin{tabular}{|c|c|c|c|}
\hline$J$ & K & Obs. & TROVE \\
\hline 2 & 0 & 2.0912 & 2.0916 \\
\hline 3 & 3 & 2.6115 & 2.6119 \\
\hline 4 & 3 & 5.3998 & 5.4006 \\
\hline 4 & 0 & 6.9707 & 6.9718 \\
\hline 5 & 3 & 8.8852 & 8.8864 \\
\hline 6 & 6 & 8.3548 & 8.3559 \\
\hline 6 & 3 & 13.0675 & 13.0694 \\
\hline 7 & 6 & 13.2342 & 13.2360 \\
\hline 7 & 3 & 17.9467 & 17.9493 \\
\hline 8 & 6 & 18.8106 & 18.8132 \\
\hline 8 & 3 & 23.5228 & 23.5263 \\
\hline 8 & 0 & 25.0935 & 25.0972 \\
\hline 9 & 9 & 17.2297 & 17.2319 \\
\hline 9 & 6 & 25.0838 & 25.0874 \\
\hline 9 & 3 & 29.7958 & 29.8002 \\
\hline 10 & 10 & 17.2297 & 17.2319 \\
\hline 10 & 9 & 24.2002 & 24.2035 \\
\hline 10 & 6 & 32.0539 & 32.0584 \\
\hline 10 & 3 & 36.7655 & 36.7709 \\
\hline 10 & 0 & 38.3360 & 38.3417 \\
\hline 20 & 18 & 89.8252 & 89.8372 \\
\hline 20 & 15 & 107.0973 & 107.1122 \\
\hline 20 & 12 & 121.2253 & 121.2425 \\
\hline 20 & 9 & 132.2114 & 132.2304 \\
\hline 20 & 6 & 140.0574 & 140.0777 \\
\hline 20 & 3 & 144.7645 & 144.7856 \\
\hline 20 & 0 & 146.3334 & 146.3548 \\
\hline 80 & 78 & 1195.6589 & 1195.8085 \\
\hline
\end{tabular}

\section{RO-VIBRATIONAL CALCULATIONS}

\section{A. Basis set convergence and Hamiltonian optimisation}

Ro-vibrational calculations were performed with the program TROVE [33] adapted to work in the $\mathcal{D}_{3 \mathrm{~h}}(\mathrm{M})$ molecular permutation-inversion group appropriate for $\mathrm{SO}_{3}$. In order to achieve results of high accuracy as well as minimising the requirement for computational 
resources, it is necessary to optimise the size of the Hamiltonian matrix. This involves preliminary truncation of the basis set, as well as limiting the order of both the kinetic and potential components of the Hamiltonian expansion. TROVE employs a polyad number truncation which controls the size of the basis set. For $\mathrm{SO}_{3}$ the polyad number is given by

$$
P=2\left(n_{1}+n_{2}+n_{3}\right)+n_{4}+n_{5}+\frac{n_{6}}{2},
$$

where $n_{i}$ are the quanta associated with $1 \mathrm{D}$ basis functions, $\phi_{i}$, whose product gives our vibrational basis set [33]. Each of these basis functions is associated with an internal coordinate $\xi_{i}$, and only functions for which $P \leq P_{\max }$ are included in the primitive basis set. Initial tests were carried out to measure the degree of convergence using different values for $P_{\max }$, and the orders of the kinetic and potential energy expansions. In this work we use a kinetic energy expansion of order 4, and a potential energy expansion of order 8; using a kinetic energy expansion order of 6 requires a more expensive calculation where convergence is already observed to within $0.001 \mathrm{~cm}^{-1}$ when expanding to fourth order. In the present study, we find that the convergence is more sensitive to $P_{\max }$, and we obtain convergence to within $0.1 \mathrm{~cm}^{-1}$ when $P_{\max }$ is 12 or 14 (see table III), therefore we use a basis set based on $P_{\max }=12$.

As well as using the polyad number to truncate the size of the basis, we employ a further truncation technique by specifying an upper limit for the eigenvalue calculations, i.e. construct the basis set such that it provides energy values up to a limit of $E_{\max }$. This is based on an estimation whereby eigenvalues of our 1D basis functions are summed together before they are considered for matrix element calculations, and the active space is constructed using basis functions whose eigenvalues sum together to have $E \leq E_{\max }$. For the present study we use $E_{\max } / h c=10000 \mathrm{~cm}^{-1}$.

These precautions are particularly important for the $\mathrm{SO}_{3}$ molecule, as its larger mass (compared to, for example, $\mathrm{XH}_{3}$ systems) gives rise to small rotational constants, which in turn requires calculations up to high $J$ value to ensure adequate coverage of transitions for a given temperature. This means that any unnecessary basis functions will prove computationally expensive. In addition to this basis set minimisation, we can reduce the size of the Hamiltonian further by making use of group theory. $\mathrm{SO}_{3}$ has $\mathcal{D}_{3 \mathrm{~h}}(\mathrm{M})$ molecular group symmetry and the spin-0 Bosons which make up the constituent atoms of this molecule allow the molecular ro-vibrational wavefunctions to exhibit the symmetry of only two of the six 
TABLE II: Convergence of basis set viewed for some vibrational band centres $\left(\mathrm{cm}^{-1}\right)$ for ${ }^{32} \mathrm{~S}^{16} \mathrm{O}_{3}$.

\begin{tabular}{lrrrr}
\hline \hline & Obs. [8] & $P_{\max }=10$ & $P_{\max }=12$ & $P_{\max }=14$ \\
\hline$\nu_{1}$ & 1064.92 & 1065.83 & 1065.75 & 1065.74 \\
$\nu_{2}$ & 497.57 & 498.48 & 498.48 & 498.48 \\
$\nu_{3}$ & 1391.52 & 1387.63 & 1387.45 & 1387.43 \\
$\nu_{4}$ & 530.09 & 528.61 & 528.59 & 528.58 \\
\hline $2 \nu_{3}\left(l_{3}=0\right)$ & 2766.40 & 2759.61 & 2759.12 & 2758.75 \\
$2 \nu_{3}\left(l_{3}=2\right)$ & 2777.87 & 2770.70 & 2770.29 & 2769.95 \\
\hline $2 \nu_{2}$ & 995.02 & 995.43 & 995.35 & 995.35 \\
$2 \nu_{4}\left(l_{4}=0\right)$ & 1059.81 & 1057.10 & 1056.50 & 1056.44 \\
$2 \nu_{4}\left(l_{4}=2\right)$ & 1060.45 & 1057.86 & 1057.38 & 1057.33 \\
$\nu_{2}+\nu_{4}\left(l_{4}=1\right)$ & 1027.90 & 1027.58 & 1027.35 & 1027.33 \\
\hline$\nu_{1}+\nu_{4}\left(l_{4}=1\right)$ & 1593.69 & 1593.82 & 1593.36 & 1593.30 \\
$3 \nu_{4}\left(l_{4}=1\right)$ & 1589.81 & 1587.64 & 1586.46 & 1586.30 \\
$3 \nu_{4}\left(l_{4}=3\right)$ & 1591.10 & 1587.61 & 1586.43 & 1586.27 \\
$\nu_{1}+\nu_{2}$ & 1560.60 & 1565.51 & 1565.33 & 1565.32 \\
$\nu_{2}+2 \nu_{4}\left(l_{4}=0\right)$ & 1557.88 & 1556.38 & 1555.59 & 1555.47 \\
$\nu_{2}+2 \nu_{4}\left(l_{4}=2\right)$ & 1558.52 & 1557.12 & 1556.45 & 1556.37 \\
$2 \nu_{2}+\nu_{4}\left(l_{4}=1\right)$ & 1525.61 & 1524.81 & 1524.48 & 1524.46 \\
$3 \nu_{2}$ & 1492.35 & 1449.81 & 1490.76 & 1490.76 \\
$\nu_{2}+\nu_{3}\left(l_{3}=1\right)$ & 1884.57 & 1881.82 & 1881.53 & 1881.51 \\
$3 \nu_{3}\left(l_{3}=1\right)$ & 4136.39 & 4138.88 & 4126.78 & 4125.92 \\
\hline \hline & & & & \\
\hline
\end{tabular}

irreducible representations of this group in order to satisfy the Pauli Principle; namely the $\mathrm{A}_{1}^{\prime}$ and $\mathrm{A}_{1}^{\prime \prime}$ representations. This reduces both the number of Hamiltonian matrices we need to consider and, since $E$ symmetry Hamiltonian matrices are larger, their size.

The two other factors which are important in our spectral calculations are i) the wavenumber range of the desired synthetic spectrum, and ii) the temperature at which we wish to simulate it. The quality of a computed spectrum will become sensitive to $E_{\max }$ as the temperature increases; we need to ensure that we calculate all energy states that are significantly 
populated for the given temperature. This can be checked using the temperature-dependent partition function:

$$
Q=\sum_{i} g_{i} \exp \left(-E_{i} / k T\right)
$$

where $g_{i}$ is the total degeneracy of the ro-vibrational state $i$ with energy $E_{i}$, with the sum running over all energies at the absolute temperature $T$, and $k$ is Boltzmann's constant. The total degeneracy is given by $(2 J+1)$ times the nuclear spin degeneracy, which for the present case of ${ }^{32} \mathrm{~S}^{16} \mathrm{O}_{3}$ is simply 1 for both the $\mathrm{A}_{1}^{\prime}$ and $\mathrm{A}_{1}^{\prime \prime}$ symmetries, given that the nuclear spin of ${ }^{16} \mathrm{O}$ and ${ }^{32} \mathrm{~S}$ are zero. For a given temperature, we can determine the contribution of various states to the value of $Q$. We can then check that $Q$ converges to a specific value as $E_{i}$ tends to infinity; as $T$ increases we require a greater coverage of higher-lying energy states. For $T=298.15 \mathrm{~K}$ we find that $Q$ converges to better than $1 \%$ at $J=85$, with a value of $Q=8089.262$. Therefore calculations spanning all $J$ 's up to 85 should be sufficient for simulating spectra at this temperature. Figure 1 shows the value of $Q$ as a function of all energy levels having $J$ quantum number up to a maximum value, $J_{\max }$, at an absolute temperature of $T=298.15 \mathrm{~K}$. As we include energies in the summation for increasing values of $J$ we see that the associated energy levels contribute less and less to the value of $Q$, until it converges to a limit.

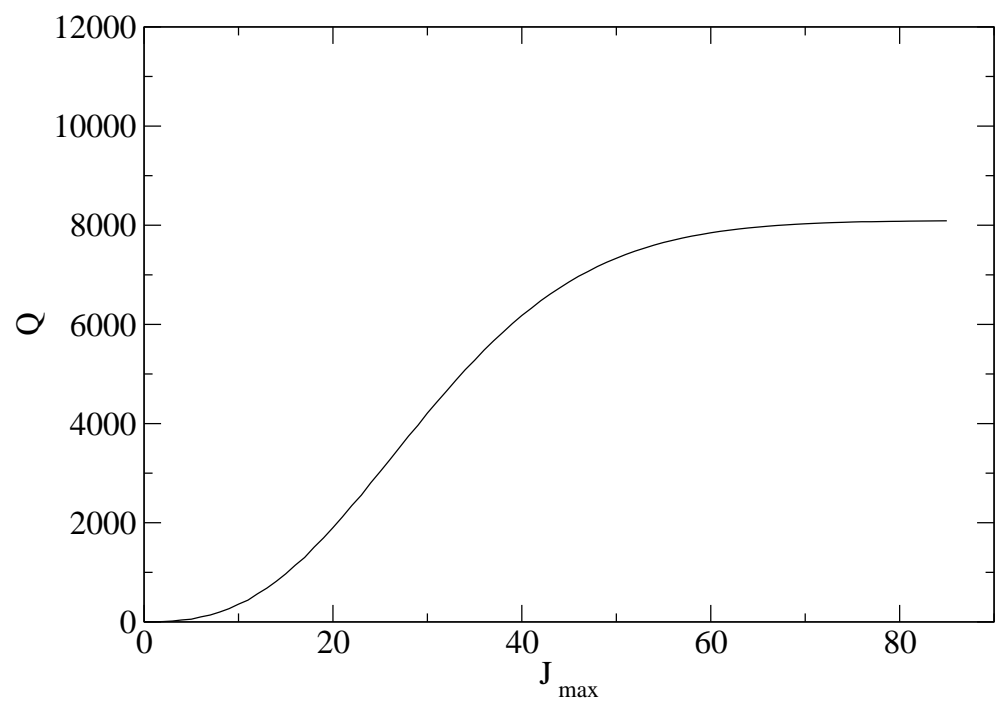

FIG. 1: Convergence of partition function for different values of $J_{\max }$.

An $a b$ initio PES was previously published by Martin based on coupled cluster meth- 
ods [14]. As an intial test of the TROVE procedure we used the quartic force field by Martin to compute fundamental term values, and to test our convergence procedures, using a polyad truncation scheme of $P_{\max }=16$. We found discrepancies between our results and the values published, particularly in the value of the $\nu_{2}$ fundamental term value. We made a substitution to the symmetry-adapted force constant $F_{22}$ associated with this vibration, taking a scaled value from a previously published force-field [13], which resulted in a fair improvement. Table IIIlustrates the differences between the values and our final converged result for the fundamental vibrations.

TABLE III: A comparison of the fundamental term values $\left(\mathrm{cm}^{-1}\right)$ between this work and Martin [14] (computed with TROVE).

\begin{tabular}{rrrrr}
\hline \hline & Obs. [8] & Martin & Martin $F_{22}$ & This work \\
\hline$\nu_{1}$ & 1064.92 & 1063.36 & 1064.22 & 1065.75 \\
$\nu_{2}$ & 497.57 & 428.36 & 487.10 & 498.48 \\
$\nu_{3}$ & 1391.52 & 1386.81 & 1386.85 & 1387.45 \\
$\nu_{4}$ & 530.09 & 527.35 & 527.32 & 528.59 \\
\hline \hline
\end{tabular}

Martin, with a substituted $F_{22}$ value [13].

\section{B. Intensity simulations}

The simulation of absorption spectra can be broken down into two main parts: first, the eigenfunctions and eigenvalues of the numerically constructed Hamiltonian matrix are calculated by a diagonalisation procedure; second, these eigenfunctions are used to compute transition dipoles, line strengthes $S$, Einstein $A$ coefficients and intensities $I$ for allowed transitions. For ${ }^{32} \mathrm{~S}^{16} \mathrm{O}_{3}$, the rigorous selection rules determining allowed electric dipole transitions are $\Delta J=J^{\prime}-J^{\prime \prime}=0, \pm 1\left(J^{\prime \prime}+J^{\prime} \geq 1\right)$, and symmetry selection rules $A_{1}^{\prime} \leftrightarrow A_{1}^{\prime \prime}$. The intensity of a transition between given states is given by the formula

$$
I(f \leftarrow i)=\frac{8 \pi^{3} N_{A} \nu_{i f}}{\left(4 \pi \epsilon_{0}\right) 3 h c} \frac{e^{-E_{i} / k T}}{Q(T)} \times\left[1-\exp \left(\frac{-h c \nu_{i f}}{k T}\right)\right] S(f \leftarrow i),
$$

where $I(f \leftarrow i)$ is the transition intensity for a transition from state $i$ with energy $E_{i}$ to state $f$ with energy $E_{f}$, with $h c \nu_{i f}=E_{f}-E_{i} . Q$ is the partition function. The value of 
$Q$ must be converged for the absolute temperature $T$, and with respect to the wavenumber range in which we wish to simulate our spectrum. $S(f \leftarrow i)$ is the line strength, which is defined by the following integration:

$$
S(f \leftarrow i)=g_{\mathrm{ns}} \sum_{m_{f}, m_{i}} \sum_{X, Y, Z}\left|\left\langle\Phi_{\mathrm{rv}}^{(f)}\left|\mu_{A}\right| \Phi_{\mathrm{rv}}^{(i)}\right\rangle\right|^{2}
$$

for a transition between initial state $i$ with rovibrational wavefunction $\Phi_{\mathrm{rv}}^{(i)}$ and final state $f$ with wavefunction $\Phi_{\mathrm{rv}}^{(f)}$. Here, $g_{\mathrm{ns}}$ is the nuclear spin statistical weight factor, and $\mu_{A}$ is the electronically averaged component of the molecular dipole moment along the space-fixed axis $A=X, Y, Z$. The quantum numbers $m_{i}$ and $m_{f}$ are projections of the total angular momentum $J$ (in units of $\hbar$ ) on the laboratory fixed $Z$ axis, for the initial and final states, respectively.

Maki et al. [5-11] reported an extensive high-resolution study of a number of fundamental, combination and overtone bands of ${ }^{32} \mathrm{~S}^{16} \mathrm{O}_{3}$. Their principle aim was to obtain accurate wavenumber measurements, but relative intensities were also measured. In the present work we convert these data into absolute intensities by normalizing to the theoretical intensities obtained with TROVE at $T=298.15 \mathrm{~K}$ as described below.

The measurements available to us cover three spectral regions: $405-708 \mathrm{~cm}^{-1}$ (focusing on $\nu_{2}, \nu_{4}, 2 \nu_{2}-\nu_{2}, \nu_{2}+\nu_{4}-\nu_{2}, \nu_{2}+\nu_{4}-\nu_{4}, \nu_{1}-\nu_{4}, 2 \nu_{4}^{\left(l_{4}=0\right)}-\nu_{4}$ and $\left.2 \nu_{4}^{\left(l_{4}=2\right)}-\nu_{4}\right), 1200-1680 \mathrm{~cm}^{-1}\left(\nu_{3}\right)$, and $2500-3280 \mathrm{~cm}^{-1}\left(2 \nu_{3}^{\left(l_{3}=2\right)}\right)$. Each measurement was made at different values of pressure. For the 405-708 $\mathrm{cm}^{-1}$ window, the measurements were performed at 0.409 and 2.04 Torr, and for the $\nu_{3}$ measurements between the $1200-1680 \mathrm{~cm}^{-1}$ window 0.16 Torr and 0.7 Torr was used. 560 lines were measured at 0.7 Torr, however 439 of these had relative intensity values which were negative. We therefore did not use this higher pressure measurement at all.

In Table IV we compare the numbers of lines identified in each measurement to the numbers of lines computed using TROVE. The latter numbers are the subject of the following selection criteria: $J \leq 85$, intensity cut-off, $I(f \leftarrow i)>10^{-34} \mathrm{~cm} /$ molecule, and the wavenumber window, $0-4000 \mathrm{~cm}^{-1}$. Experimental lines with negative relative intensities were left out of the analysis.

To normalize the experimental intensities, the experimental relative data from each spectral window and each different pressure were scaled to match the theoretical values computed at $T=298.15 \mathrm{~K}$. The scaling factors obtained through a minimization pro- 
cedure using all selected experimental lines with non-zero intensity (see Table V]) are $6.571 \times 10^{-21} \mathrm{~cm} /$ molecule (405-708 $\mathrm{cm}^{-1}, 0.409$ Torr), $1.838 \times 10^{-21} \mathrm{~cm} /$ molecule (405-708 $\mathrm{cm}^{-1}, 2.04$ Torr $), 4.823 \times 10^{-20} \mathrm{~cm} /$ molecule ( $1200-1680 \mathrm{~cm}^{-1}, 0.16$ Torr $)$, and $1.328 \times 10^{-21}$ cm/molecule (2500-3280 $\mathrm{cm}^{-1}, 4.99$ Torr). With these factors the 'experimental' intensities match the theoretical values reasonably good, for example for $405-708 \mathrm{~cm}^{-1}$ the agreement is within about $7.02 \times 10^{-22} \mathrm{~cm} /$ molecule and $5.05 \times 10^{-22} \mathrm{~cm} /$ molecule, at 0.409 and 2.04 Torr, respectively.

Having the absolute intensities derived, band intensities were estimated for each experimental band as the sum of individual line intensities. In Table $\mathrm{V}$ these 'experimental' band intensities $S^{\exp }$ (cm/molecule) are compared to the theoretical values obtain by summing intensities (a) from all TROVE lines from a given window and (b) only from lines with experimental counterparts present. This was done separately for each spectral range, and each measurement pressure therein. In Table $\mathrm{V}$ these quantities are referenced to as $S_{\text {tot }}^{\text {calc }}$ and $S_{\text {red }}^{\text {calc }}$ for the 'total' and 'reduced' band intensities, respectively, and compared to $S^{\exp }$. The ratio $S_{\text {red }}^{\text {calc }}$ to $S^{\exp }$ also shown in Table $\nabla$ demonstrates the good quality of the procedure employed as well as of our dipole moment. For example at 0.409 Torr, the differences between 'experimental' and theoretical band intensities are within about $20 \%$ for all bands from the $405-708 \mathrm{~cm}^{-1}$ region with the exception of $2 \nu_{4}^{\left(l_{4}=0\right)}-\nu_{4}$ (see also discussion below). It should be stressed here that only one scaling factor for all eight bands from this window was applied at a given pressure. The difference between two theoretical band intensities

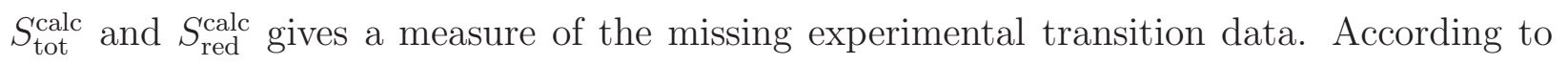
Table $\mathrm{V}$ even stronger bands miss more that $50 \%$ of the total intensity.

In Table $\nabla$ we also show theoretical values of vibrational transition moments defined as

$$
\bar{\mu}=\sqrt{\bar{\mu}_{x}^{2}+\bar{\mu}_{y}^{2}+\bar{\mu}_{z}^{2}}
$$

where

$$
\bar{\mu}_{\alpha}=\left\langle\Psi_{\mathrm{vib}}^{(i)}\left|\mu_{\alpha}\right| \Psi_{\mathrm{vib}}^{(f)}\right\rangle
$$

and $\Psi_{\text {vib }}^{(i)}$ and $\Psi_{\text {vib }}^{(f)}$ are the vibrational eigenfunctions of the 'initial' and 'final' states, respectively, variationally computed using TROVE and $\mu_{\alpha}$ is the component of the molecular dipole moment along the molecular-fixed axis $\alpha=x, y, z$.

Figure 2 presents an overview of the simulated spectrum $(T=298.15 \mathrm{~K})$ with TROVE and experimental absorption spectra of $\mathrm{SO}_{3}$ for the whole simulation range up to $4000 \mathrm{~cm}^{-1}$. 
It reveals the gaps and limitations of the available experimental data. Our intensities based on the ab initio DMS are in very good qualitative agreement with experiment. Figure 3 shows the 'forbidden' rotational band as a stick spectrum. It should be noted that the microwave measurements from Ref. [12] do not have any intensities reported. In Figure 4 all eight bands from the $405-708 \mathrm{~cm}^{-1}$ region are combined into one graph for each pressure to illustrate the quality of the corresponding experimental data. This figure suggests that the 0.409 Torr data are generally more reliable. This is also reflected by the ratio values $S_{\text {red }}^{\text {calc }} / S^{\exp }$ from Table $\nabla$, which are significantly closer to 1 at the lower pressure. Of the data present for two conditions we therefore place preference on scaled intensity values obtained at the lower pressure. Finally, Figure 5 presents a detailed comparison for the all bands from the three spectral regions studied in this work in the form of stick diagrams.

TABLE IV: Comparison of calculated (TROVE) and experimental [8] band centers and numbers of line transitions.

\begin{tabular}{|c|c|c|c|c|c|}
\hline Band & Obs. & Calc. & Pressure $P 1^{a}$ & Pressure $P 2^{b}$ & TROVE \\
\hline$\nu_{2}-\nu_{0}$ & 497.57 & 498.48 & 773 & 1265 & 5422 \\
\hline$\nu_{4}-\nu_{0}$ & 530.09 & 528.59 & 996 & 2052 & 12195 \\
\hline$\nu_{1}-\nu_{4}$ & 534.83 & 537.16 & 0 & 69 & 15147 \\
\hline$\nu_{2}+\nu_{4}-\nu_{2}$ & 530.33 & 528.87 & 84 & 571 & 12477 \\
\hline $2 \nu_{2}-\nu_{2}$ & 497.45 & 496.88 & 112 & 704 & 7171 \\
\hline$\nu_{2}+\nu_{4}-\nu_{4}$ & 497.81 & 498.76 & 47 & 602 & 27182 \\
\hline $2 \nu_{4}^{\left(l_{4}=2\right)}-\nu_{4}$ & 530.36 & 528.79 & 116 & 775 & 31096 \\
\hline $2 \nu_{4}^{\left(l_{4}=0\right)}-\nu_{4}$ & 529.72 & 527.91 & 39 & 455 & 13718 \\
\hline$\nu_{3}-\nu_{0}$ & 1391.52 & 1387.45 & 2014 & - & 14441 \\
\hline $2 \nu_{3}^{\left(l_{3}=2\right)}-\nu_{0}$ & 2777.87 & 2770.29 & 1527 & - & 18115 \\
\hline$\nu_{0}-\nu_{0}$ & - & - & 25 & - & 3439 \\
\hline
\end{tabular}

For the $\nu_{3}$ and $2 \nu_{3}$ bands, measurements were taken at 0.16 Torr and 4.99 Torr respectively. Pressure values are not recorded for microwave measurements [12]. The remainder are the bands within the $405-708 \mathrm{~cm}^{-1}$ window, measured at 0.409 Torr (Maki et al. [8]).

Bands within the $405-708 \mathrm{~cm}^{-1}$ window, measured at 2.04 Torr (Maki et al. [8]). 
TABLE V: Vibrational band intensities $S^{\text {exp }}, S_{\text {tot }}^{\text {calc }}, S_{\text {red }}^{\text {calc }}$ in $\mathrm{cm} / \mathrm{molecule} \times 10^{-18}$, and calculated transition moments $\bar{\mu}_{\text {if }}$ in Debye. $P_{1}$ and $P_{2}$ refer to the different pressure measurements within the same wavenumber region (see Table IV]) $. S_{\text {red }}^{\text {calc }} / S^{\exp }$ is the ratio of the theoretical reduced and total band intensities (see text). $S_{\text {tot }}^{\text {calc }}$ is the theoretical band intensity computed by summing all TROVE lines. $S^{\exp }$ is the experimental band intensity obtained from a summation over all experimental values after scaling factors applied (see text). $S_{\text {red }}^{\text {calc }}$ is the theoretical band intensity computed using only lines for which experimental counterparts exist. $N_{\text {red }}$ is the number of matched lines.

\begin{tabular}{|c|c|c|c|c|c|c|c|c|c|c|}
\hline \multirow[t]{2}{*}{ Band } & \multicolumn{2}{|c|}{$S^{\exp }$} & \multirow[t]{2}{*}{$S_{\text {tot }}^{\text {calc }}$} & \multicolumn{2}{|c|}{$S_{\text {red }}^{\text {calc }}$} & \multicolumn{2}{|c|}{$S_{\mathrm{red}}^{\text {calc }} / S^{\exp }$} & \multicolumn{2}{|c|}{$N_{\text {red }}$} & \multirow[t]{2}{*}{$\bar{\mu}_{\mathrm{if}} / D$} \\
\hline & $P_{1}$ & $P_{2}$ & & $P_{1}$ & $P_{2}$ & $P_{1}$ & $P_{2}$ & $P_{1}$ & $P_{2}$ & \\
\hline$\nu_{2}$ & 2.987 & 1.537 & 3.705 & 2.915 & 1.559 & 0.98 & 1.01 & 773 & 1265 & 0.158 \\
\hline$\nu_{4}$ & 4.258 & 3.112 & 5.949 & 4.310 & 3.149 & 1.01 & 1.01 & 995 & 2052 & 0.200 \\
\hline $2 \nu_{2}-\nu_{2}$ & 0.116 & 0.470 & 0.661 & 0.101 & 0.411 & 0.88 & 0.88 & 112 & 704 & 0.221 \\
\hline$\nu_{2}+\nu_{4}-\nu_{2}$ & 0.062 & 0.322 & 0.528 & 0.052 & 0.251 & 0.84 & 0.78 & 84 & 571 & 0.199 \\
\hline$\nu_{2}+\nu_{4}-\nu_{4}$ & 0.026 & 0.260 & 0.581 & 0.022 & 0.215 & 0.84 & 0.83 & 47 & 602 & 0.223 \\
\hline $2 \nu_{4}\left(l_{4}=2\right)-\nu_{4}$ & 0.112 & 0.589 & 0.873 & 0.102 & 0.485 & 0.91 & 0.82 & 116 & 769 & 0.283 \\
\hline $2 \nu_{4}\left(l_{4}=0\right)-\nu_{4}$ & 0.026 & 0.222 & 0.405 & 0.015 & 0.179 & 0.57 & 0.81 & 38 & 454 & 0.196 \\
\hline$\nu_{1}-\nu_{4}$ & - & 0.009 & 0.101 & - & 0.003 & - & 0.29 & - & 69 & 0.039 \\
\hline$\nu_{3}$ & 39.490 & - & 44.440 & 39.490 & - & - & - & 2014 & - & 0.321 \\
\hline $2 \nu_{3}$ & 0.093 & - & 0.119 & 0.093 & - & - & - & 1527 & - & 0.012 \\
\hline
\end{tabular}

\section{CONCLUSIONS AND DISCUSSIONS}

The misplaced theoretical bands in Figures 4 and 5 indicate that our ab initio PES of $\mathrm{SO}_{3}$ requires improvement (see also band centers in Table IV]). The theoretical $\nu_{2}$ frequencies have an rms deviation of $0.91 \mathrm{~cm}^{-1}$ when compared with the experimental data, which is relatively small compared to the deviation for $\nu_{3}$ of $4.07 \mathrm{~cm}^{-1}$. This is to be expected since our PES is purely ab initio computed at a modest level of theory. We are planning to refine this surface by fitting to all experimental data available.

Table $\square$ outlines the quality of the intensity scaling procedure, in which the relative values of the experimental intensities where converted to absolute values $(\mathrm{cm} / \mathrm{molecule})$. For the 0.409 Torr and 2.04 Torr measurements our comparisons mostly agree to within 


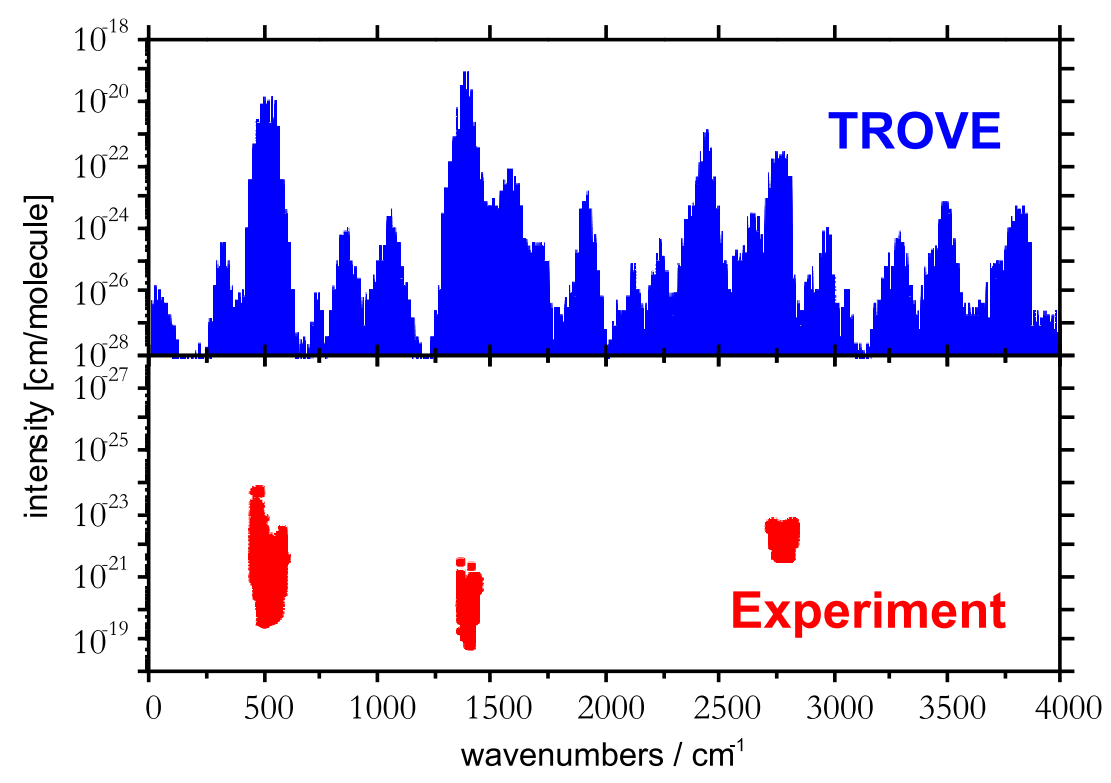

FIG. 2: Overview of the simulated absorption $(T=298.15 \mathrm{~K})$ spectrum (TROVE) of $\mathrm{SO}_{3}$ compared to experiment scaled to the theoretical intensities (see text).

$20 \%$, with the exception of the $2 \nu_{4}^{\left(l_{4}=0\right)}-\nu_{4}$ band measured at 0.409 Torr which shows nearly a $50 \%$ difference, and the $\nu_{1}-\nu_{4}$ band measured at 2.04 Torr with $80 \%$ uncertainty. The latter can probably be attributed to both the small number of lines in each case, and residual errors in the transition dipole. In the case of the $2 \nu_{4}^{\left(l_{4}=0\right)}-\nu_{4}$ band the comparison with the 2.04 Torr experiment yields a better value for $S_{\text {red }}^{\text {calc }} / S^{\exp }$ than for the 0.409 Torr measurement, which suggests that the number of lines available at 0.409 Torr is too low (see also Figure 4) The significance of the results presented in Table $\mathrm{V}$ and illustrated in Figure 4 is that these give an estimation on the quality of the $a b$ initio dipole moment surface as well as of the experimental data. Based these numbers we can place a lower estimate on the quality uncertainty for our intensities for each band, for example the experiment and theory for the $\nu_{2}$ and $\nu_{4}$ bands agree at least to within $3 \%$ for both pressure measurements, while it is only $13 \%$ for $2 \nu_{2}-\nu_{2}$, and between $17 \%-18 \%$ for the remaining bands.

Our complete room-temperature line list for $\mathrm{SO}_{3}$ containing 349348513 transitions can be accessed online at www.exomol.com in the ExoMol format described in [34]. It includes the transition energies, Einstein coefficients $A(f \leftarrow i)$, and absorption intensities estimated for $T=298.15 \mathrm{~K}$. Additionally, a list of 10878 experimental transitions with absolute intensities obtained for $T=296 \mathrm{~K}$ is included into the supplementary part of this paper in 


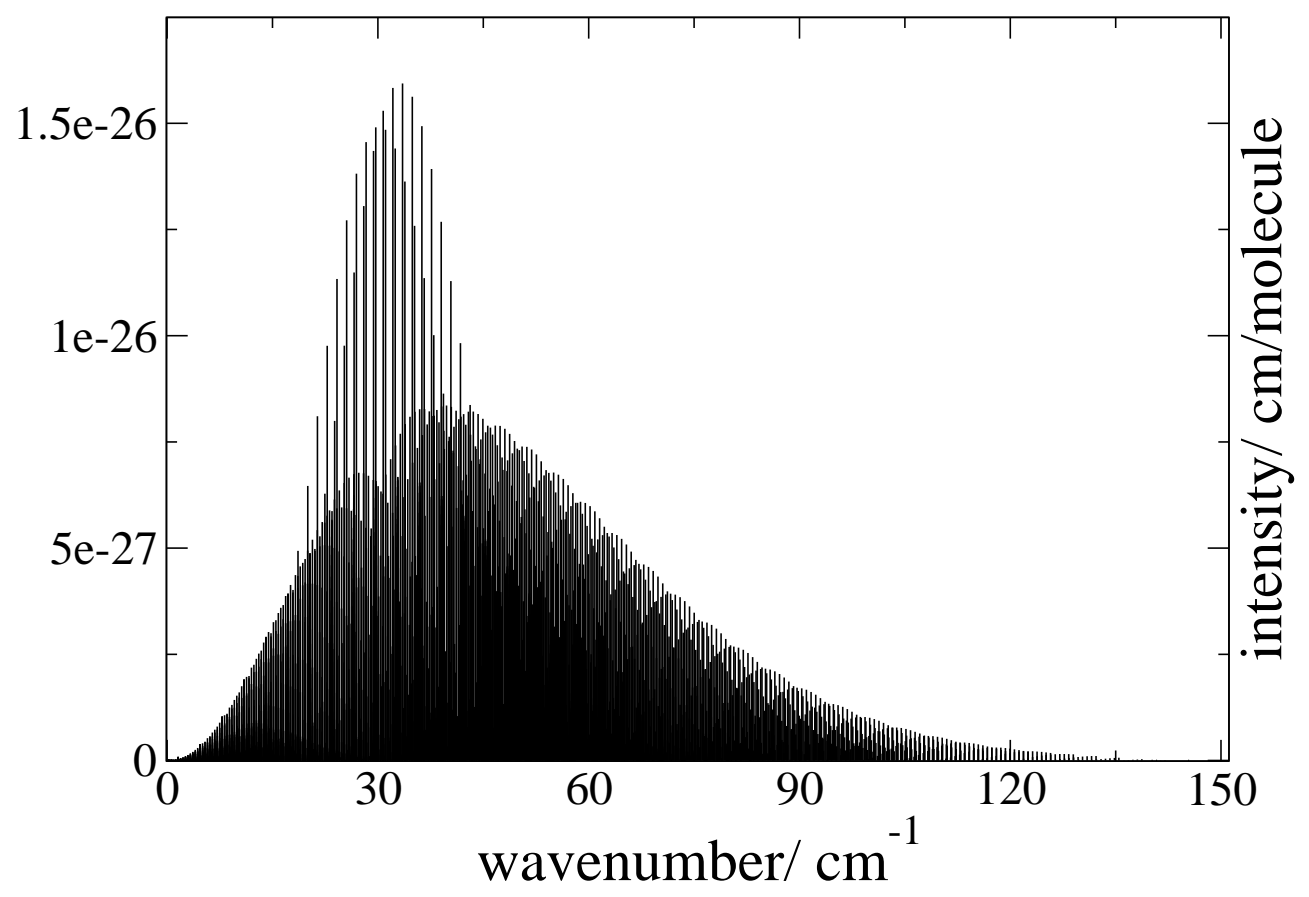

FIG. 3: Rotational absorption band computed for $T=298.15 \mathrm{~K}$, complete up to $J=85$.

a form suitable for standard atmospheric and planetary spectroscopic databases.

Our future work will be focused on the development of a hot line list for ${ }^{32} \mathrm{~S}^{16} \mathrm{O}_{3}$ for high temperature industrial applications as well as for modelling molecular opacity in atmospheres of (exo-)planets and cool stars as part of the ExoMol project [35] (see www.exomol.com).

\section{Acknowledgment}

We thank Alexander Fateev for stimulating our interest in this molecule and for many helpful discussions, and Jeff Barber for supplying his experimental result. This work was supported by grant 10442 from energinet.dk under a subcontract from the Danish Technical University and and the ERC under Advanced Investigator Project 267219. 

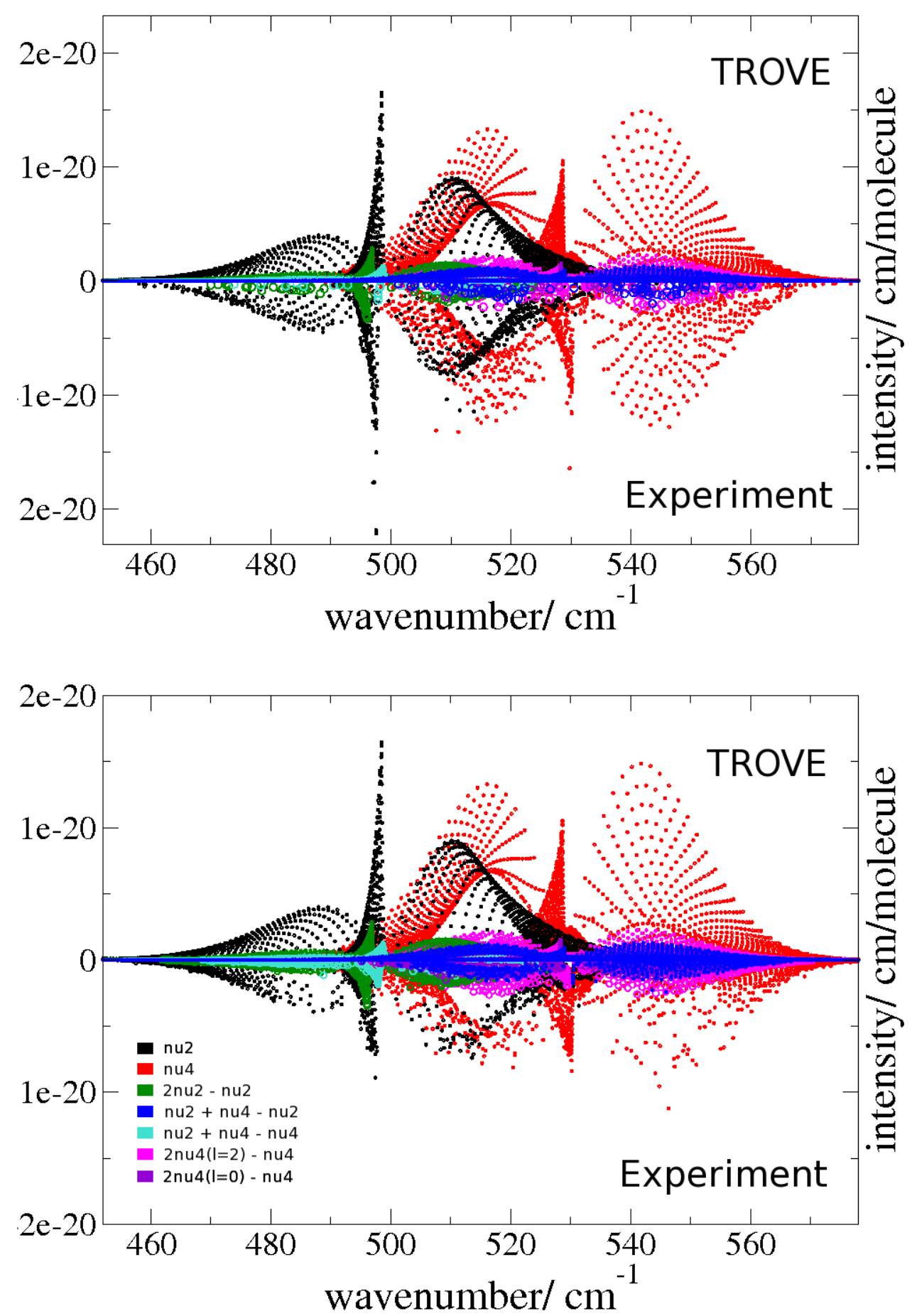

FIG. 4: Comparison plot of TROVE results and the bands of interest measured between $405-708$ $\mathrm{cm}^{-1}$ by Maki et al. , at 0.409 Torr (above) and 2.04 Torr (below). Points are enlarged in some cases for clarity. 

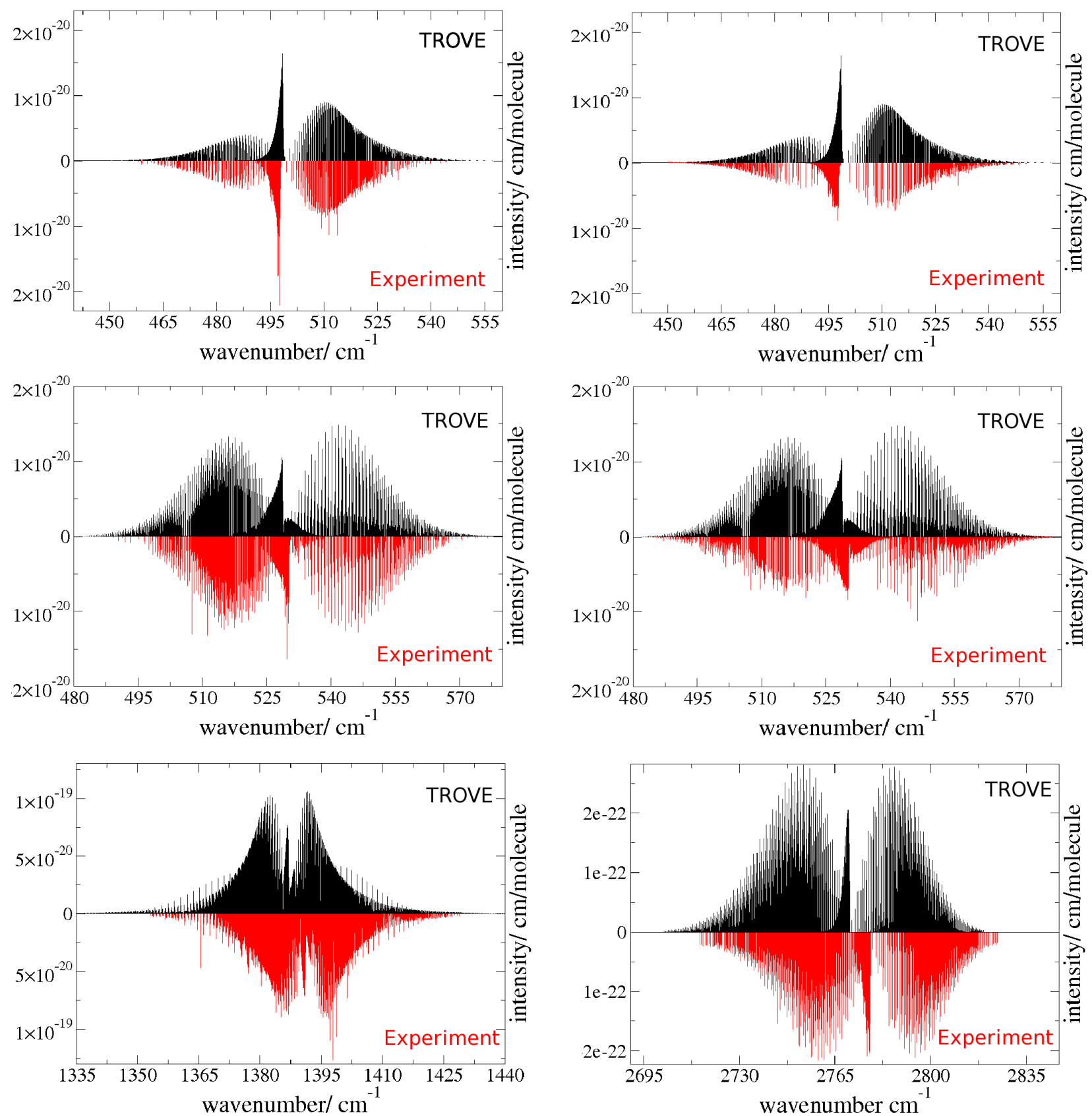

FIG. 5: Fundamental band comparisons between this work $(T=298.15 \mathrm{~K}$, complete up to $J=85)$ and Maki et al. [8]. The top and middle panels show comparisons for the $\nu_{2}$ and $\nu_{4}$ bands, respectively, at 0.409 Torr (left) and 2.04 Torr (right). The bottom panel shows a comparison for the $\nu_{3}$ band at 0.16 Torr (left) and the $2 \nu_{3}$ band at 4.99 Torr (right). 


\section{REFERENCES}

[1] W. T. Rawlins, J. M. Hensley, D. M. Sonnenfroh, D. B. Oakes and M. G. Allen, Appl. Optics, $2005,44,6635-6643$.

[2] D. Fleig, E. Vainio, K. Andersson, A. Brink, F. Johnsson and M. Hupa, Energy Fuels, 2012, 26, 5537-5549.

[3] J. P. Michaud, D. Krupitsky, J. S. Grove and B. S. Anderson, Neurotoxicology, 2005, 26, $555-563$.

[4] X. Zhang, M.-C. Liang, F. Montmessin, J.-L. Bertaux, C. Parkinson and Y. L. Yung, Nat. Geosci., 2010, 3, 834-837.

[5] A. Kaldor, A. G. Maki, A. J. Dorney and I. M. Mills, J. Mol. Spectrosc., 1973, 45, 247-252.

[6] J. Ortigoso, R. Escribano and A. G. Maki, J. Mol. Spectrosc., 1989, 138, 602-613.

[7] E. T. H. Chrysostom, N. Vulpanovici, T. Masiello, B. J, J. W. Nibler, A. Weber, A. Maki and T. A. Blake, J. Mol. Spectrosc., 2001, 210, 233-239.

[8] A. Maki, T. A. Blake, L. S. Sams, N. Vulpanovici, J. Barber, E. T. H. Chrysostom, T. Masiello, J. W. Nibler and A. Weber, J. Mol. Spectrosc., 2001, 210, 240-249.

[9] J. Barber, E. T. H. Chrysostom, T. Masiello, J. W. Nibler, A. Maki, A. Weber, T. A. Blake and R. L. Sams, J. Mol. Spectrosc., 2002, 216, 105-112.

[10] S. W. Sharpe, T. A. Blake, R. L. Sams, A. Maki, T. Masiello, J. Barber, N. Vulpanovici, J. W. Nibler and A. Weber, J. Mol. Spectrosc., 2003, 222, 142-152.

[11] A. Maki, T. A. Blake, R. L. Sams, J. Frieh, J. Barber, T. Masiello, E. T. H. Chrysostom, J. W. Nibler and A. Weber, J. Mol. Spectrosc., 2004, 225, 109-122.

[12] V. Meyer, D. H. Sutter and H. Dreizler, Z. Naturforsch. A, 1991, 46, 710-714.

[13] A. J. Dorney, A. R. Hoy and I. M. Mills, J. Mol. Spectrosc., 1973, 45, 253-260.

[14] J. M. L. Martin, Spectrochimica Acta A, 1999, 55, 709-718.

[15] L. S. Rothman, I. E. Gordon, A. Barbe, D. C. Benner, P. F. Bernath, M. Birk, V. Boudon, L. R. Brown, A. Campargue, J. P. Champion, K. Chance, L. H. Coudert, V. Dana, V. M. Devi, S. Fally, J. M. Flaud, R. R. Gamache, A. Goldman, D. Jacquemart, I. Kleiner, N. Lacome, W. J. Lafferty, J. Y. Mandin, S. T. Massie, S. N. Mikhailenko, C. E. Miller, N. Moazzen- 
Ahmadi, O. V. Naumenko, A. V. Nikitin, J. Orphal, V. I. Perevalov, A. Perrin, A. PredoiCross, C. P. Rinsland, M. Rotger, M. Simeckova, M. A. H. Smith, K. Sung, S. A. Tashkun, J. Tennyson, R. A. Toth, A. C. Vandaele and J. Vander Auwera, J. Quant. Spectrosc. Radiat. Transf., 2009, 110, 533-572.

[16] N. Jacquinet-Husson, L. Crepeau, R. Armante, C. Boutammine, A. Chédin, N. A. Scott, C. Crevoisier, V. Capelle, C. Boone, N. Poulet-Crovisier, A. Barbe, A. Campargue, D. C. Benner, Y. Benilan, B. Bézard, V. Boudon, L. R. Brown, L. H. Coudert, A. Coustenis, V. Dana, V. M. Devi, S. Fally, A. Fayt, J.-M. Flaud, A. Goldman, M. Herman, G. J. Harris, D. Jacquemart, A. Jolly, I. Kleiner, A. Kleinböhl, F. Kwabia-Tchana, N. Lavrentieva, N. Lacome, L.-H. Xu, O. M. Lyulin, J.-Y. Mandin, A. Maki, S. Mikhailenko, C. E. Miller, T. Mishina, N. Moazzen-Ahmadi, H. S. P. Müller, A. Nikitin, J. Orphal, V. Perevalov, A. Perrin, D. T. Petkie, A. Predoi-Cross, C. P. Rinsland, J. J. Remedios, M. Rotger, M. A. H. Smith, K. Sung, S. Tashkun, J. Tennyson, R. A. Toth, A.-C. Vandaele and J. Vander Auwera, J. Quant. Spectrosc. Radiat. Transf., 2011, 112, 2395-2445.

[17] T. Adler, G. Knizia and H.-J. Werner, J. Chem. Phys., 2007, 127, 221106.

[18] K. E. Yousaf and K. A. Peterson, Chem. Phys. Lett., 2009, 476, 303.

[19] K. E. Yousaf and K. A. Peterson, J. Chem. Phys., 2008, 129, 184108).

[20] F. Weigend, Phys. Chem. Chem. Phys., 2002, 4, 4285-4291.

[21] C. Hättig, Phys. Chem. Chem. Phys., 2005, 7, 59-66.

[22] H. J. Werner, P. J. Knowles, R. Lindh, F. R. Manby, M. Schütz et al., MOLPRO, a package of ab initio programs, 2010, see http://www.molpro.net/.

[23] S. N. Yurchenko, M. Carvajalz, P. Jensen, H. Lin, J. J. Zheng and W. Thiel, Mol. Phys., 2005, 103, 359-378.

[24] H. Lin, W. Thiel, S. N. Yurchenko, M. Carvajal and P. Jensen, J. Chem. Phys., 2002, 117, $11265-11276$.

[25] S. N. Yurchenko, W. Thiel and P. Jensen, J. Mol. Spectrosc., 2006, 240, 174-187.

[26] R. I. Ovsyannikov, W. Thiel, S. N. Yurchenko, M. Carvajal and P. Jensen, J. Mol. Spectrosc., 2008, 252, 121-128.

[27] S. N. Yurchenko, W. Thiel, M. Carvajal and P. Jensen, Chem. Phys., 2008, 346, 146-159.

[28] H. Partridge and D. W. Schwenke, J. Chem. Phys., 1997, 106, 4618-4639.

[29] S. N. Yurchenko, R. J. Barber and J. Tennyson, Mon. Not. R. Astr. Soc., 2011, 413, $1828-$ 
1834 .

[30] A. E. Lynas-Gray, S. Miller and J. Tennyson, J. Mol. Spectrosc., 1995, 169, 458-467.

[31] L. Lodi, J. Tennyson and O. L. Polyansky, J. Chem. Phys., 2011, 135, 034113.

[32] S. N. Yurchenko, J. Tennyson, R. J. Barber and W. Thiel, J. Mol. Spectrosc., to be submitted, 2013.

[33] S. N. Yurchenko, W. Thiel and P. Jensen, J. Mol. Spectrosc., 2007, 245, 126-140.

[34] J. Tennyson, C. Hill and S. N. Yurchenko, 2013.

[35] J. Tennyson and S. N. Yurchenko, Mon. Not. R. Astr. Soc., 2012, 425, 21-33. 


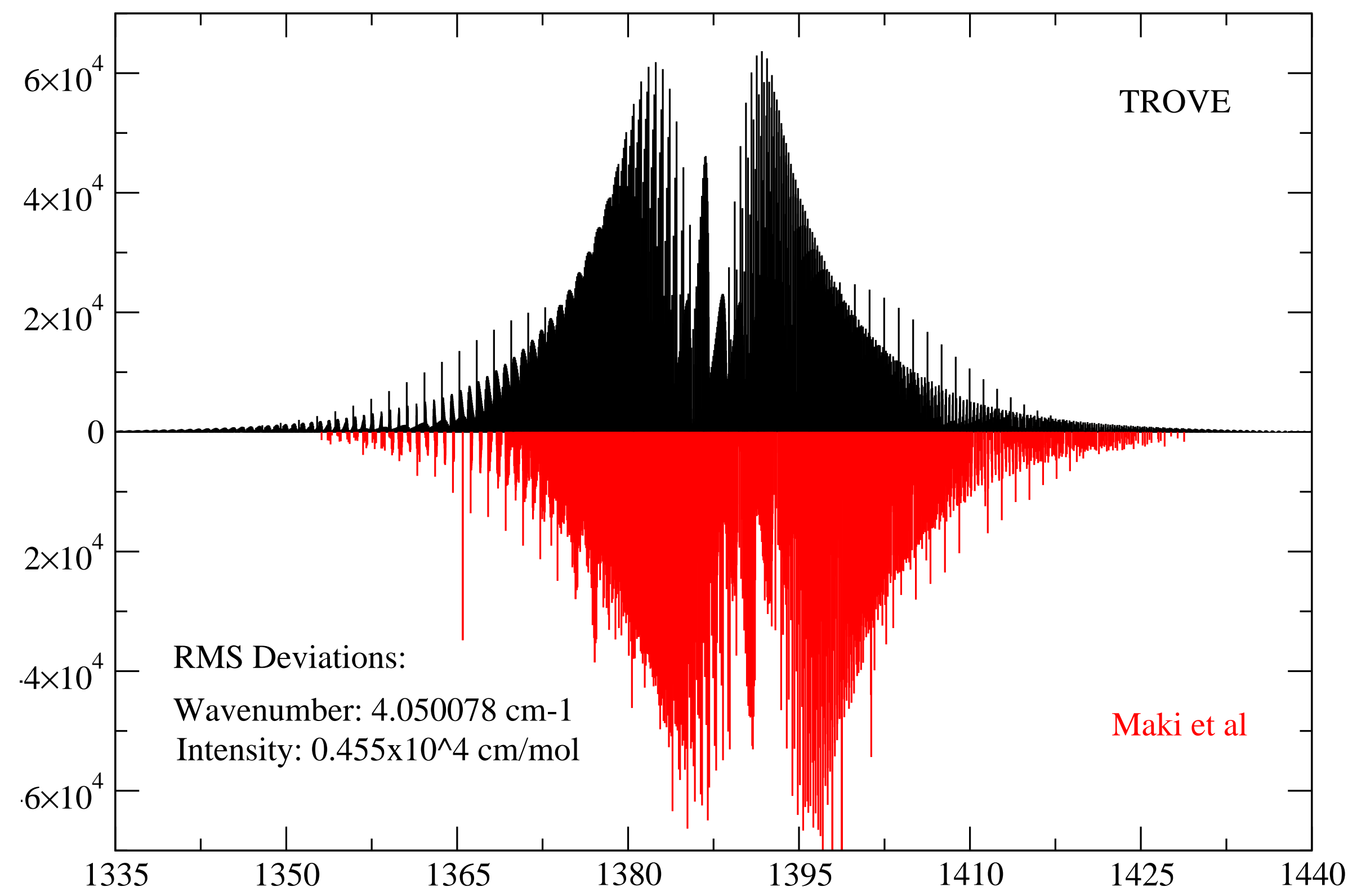




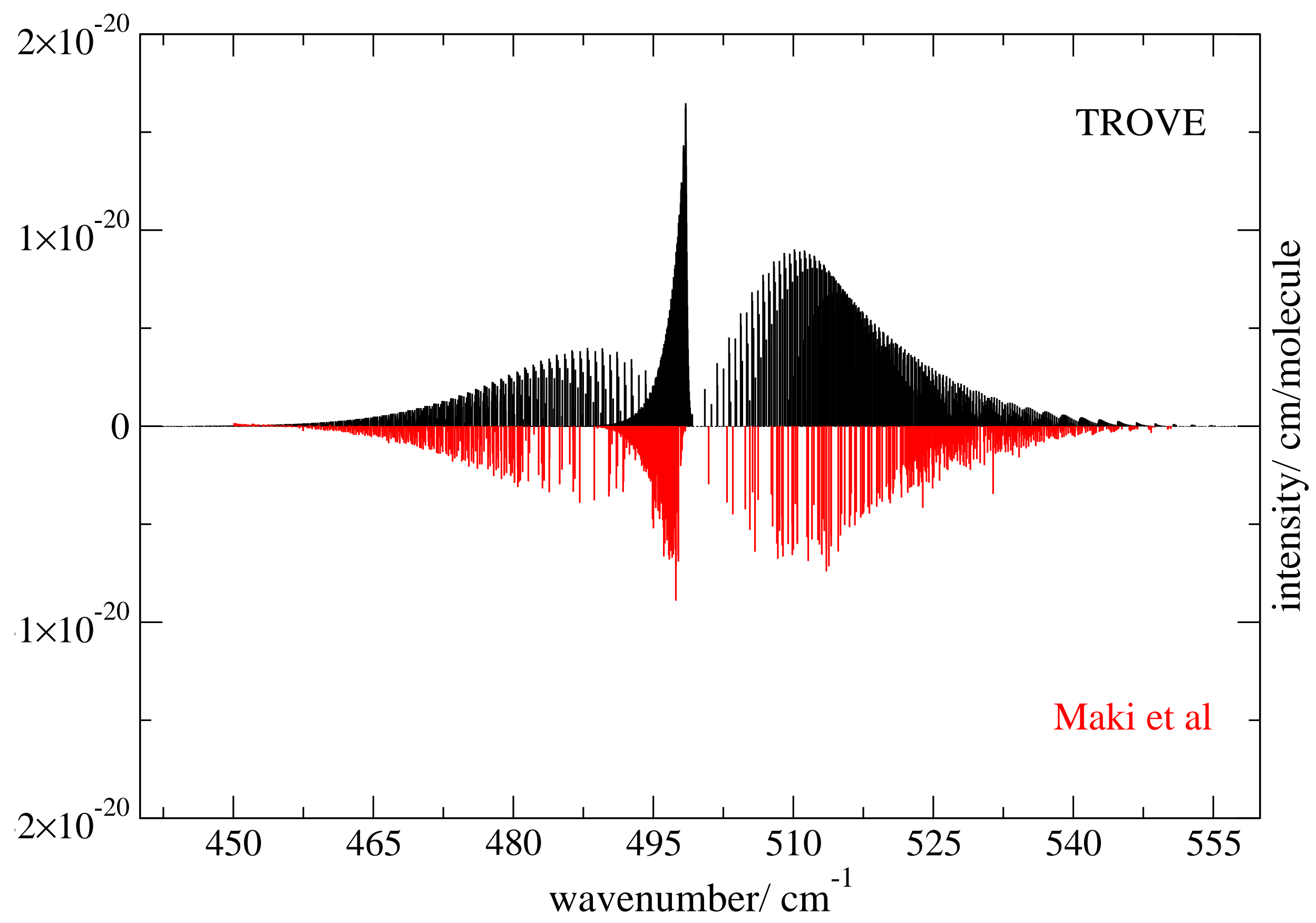




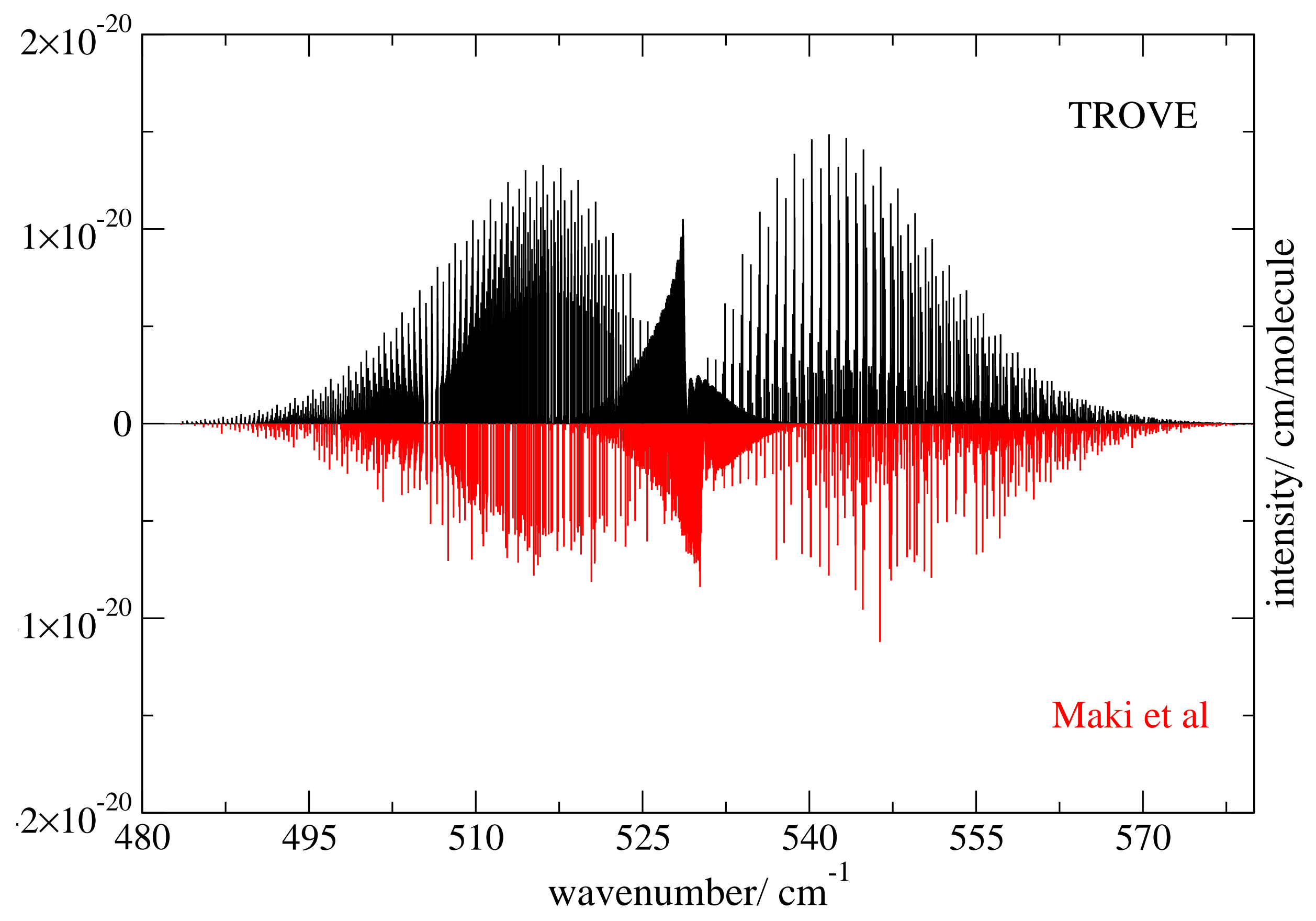




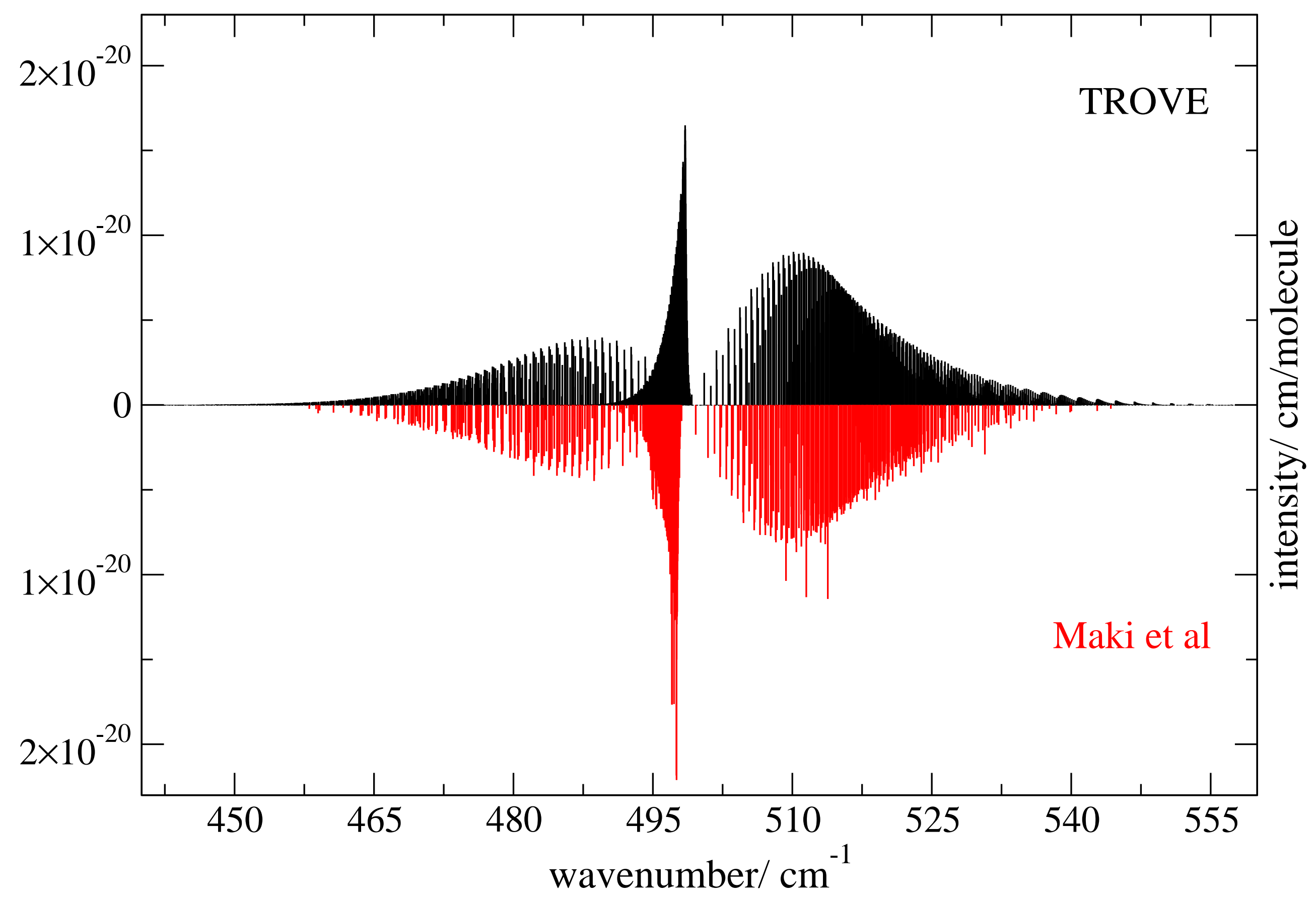




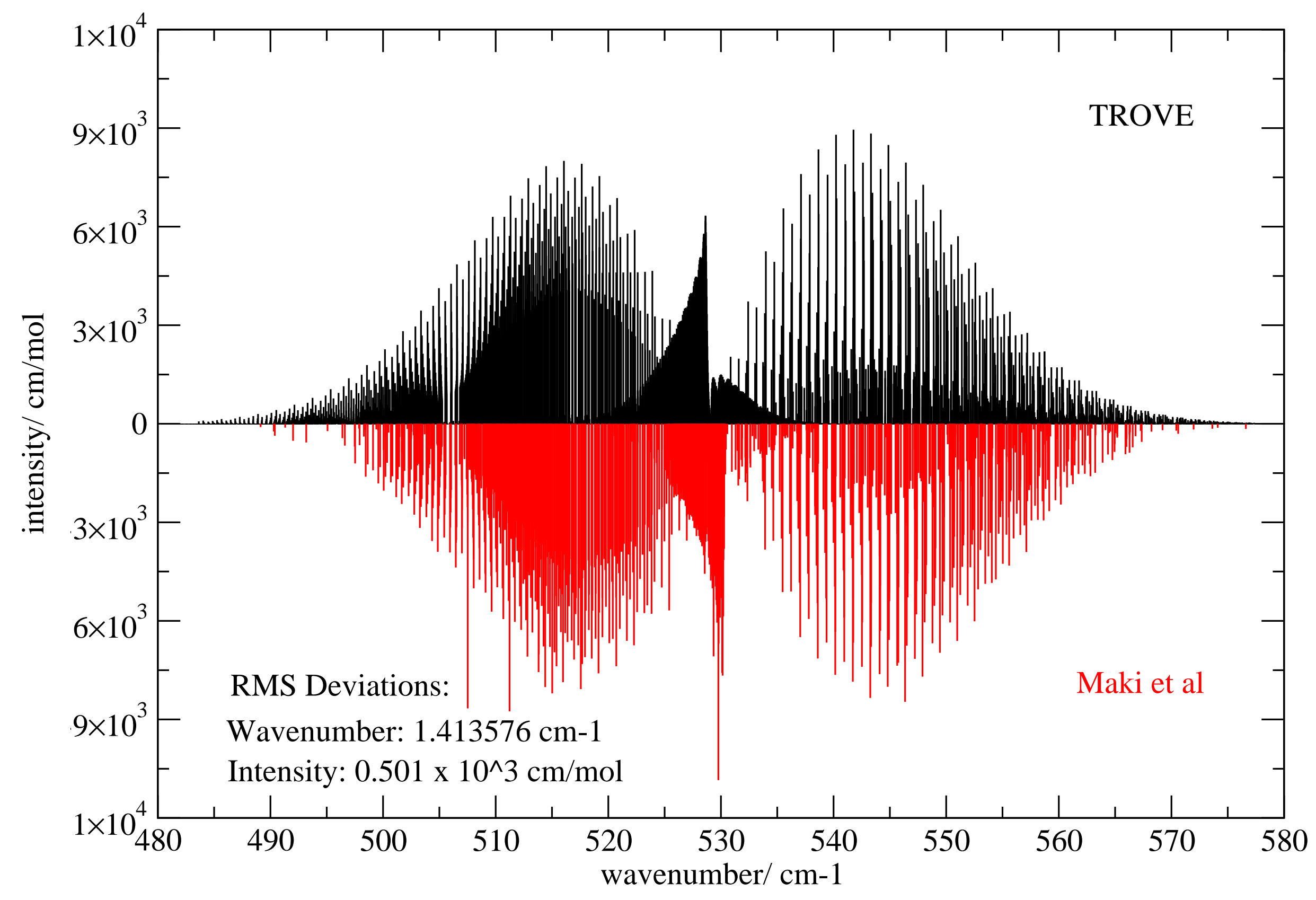

